\title{
Fronteras y contrabando: etnografía del comercio de mercancías-imitaciones en la Espacialidad Fronteriza de Atacama-Lípez (EFAL) (Chile y Bolivia)
}

Borders and smuggling: ethnography of merchandise trade-imitations in the Border Space of Atacama-Lípez (EFAL) (Chile and Bolivia)

José Miguel Muñoz Valenzuela*

*Universidad Católica del Norte - San Pedro de Atacama, Antofagasta, Chile Doctorando en Antropología (becario CONICYT) jose.munoz.va@gmail.com https://orcid.org/0000-0002-6249-2405 


\title{
Resumen
}

El texto tiene por objeto visibilizar formas y procesos contemporáneos relativos a la circulación de personas y cosas que participan del comercio y contrabando de mercancías-imitaciones provenientes de Asia Oriental en la EFAL (Chile y Bolivia). Históricamente, tales dinámicas se vinculan con el ascenso e incorporación económica de grupos sociales marginados debido a la impronta colonial del capitalismo, mercado y estado en nuestra Región, siendo sancionadas simultáneamente con un carácter difuso y/o criminal por evadir la fiscalidad estatal y las leyes internacionales de copyright. Se observa que tales prácticas [re]producen nuevas geografías a partir de las articulaciones entre las economías asiáticas y Suramérica desde 1970, permitiendo, por una parte, que las poblaciones locales intervengan en parte del control de los símbolos asociados a las cosas que se movilizan por ésta; y una serie de transformaciones y reordenamientos espaciales, sociopolíticos, cognitivos y culturales multiescalares, por la otra.

Palabras clave: fronteras; contrabando; imitaciones; Bolivia y Chile.

\begin{abstract}
Abtsract
The purpose of the text is to visualize contemporary forms and processes related to the circulation of people and things that participate in the trade and smuggling of merchandise-imitations from East Asia into the EFAL (Chile and Bolivia). Historically, such dynamics are linked to the rise and economic incorporation of marginalized social groups due to the colonial imprint of capitalism, market and state in our Region, being sanctioned simultaneously with a diffuse and/or criminal character for evading state taxation and laws international copyright It is observed that such practices [re] produce new geographies from the articulations between the Asian mega-economies and South America since 1970, allowing, on the one hand, that local populations intervene in part to control the symbols associated with the things that are mobilized for this; and a series of transformations and spatial, sociopolitical, cognitive and multiscalar cultural rearrangements, on the other.
\end{abstract}

Keywords: borders: smuggling; imitations; Bolivia and Chile. 


\section{Introducción $^{1}$}

La "ira de los productos falsificados", ${ }^{2}$ como indica Lin Yi-Chieh (2011), es una de las metáforas más asertivas para representar tanto material como simbólicamente la magnitud e intensidad contemporáneas que tienen las mercancías-imitaciones en los fenómenos relativos en las economías populares y específicamente al contrabando, en la situación contemporánea del neoliberalismo global y de las nuevas geografías económicas resultantes de las articulaciones que las economías de Asia Oriental han establecido con Suramérica a contar de la segunda mitad del siglo XX. Ira, porque tales fenómenos se observan no solo como una actividad descontrolada sino impredecible, incluso en la forma en que ésta se espacializa.

El empleo de esta metáfora respecto de la fuerza que sostiene tal movilidad de personas y cosas que inundan mercados nutridos a partir del contrabando en la Región, cuyo stock y variedad ha evolucionado notoriamente en las últimas décadas, ampliándose a todos los géneros y tipos -incluso inventando nuevos (Han, 2016, p. 76)-, y que entronca laboralmente a miles de personas, permite observar un fenómeno económico global impulsivo, que no ha sido abordado adecuadamente por la ciencia social en tanto representa una acción criminal, vale decir, una práctica penalizada por el solo hecho de haber sido sancionada de esa manera por los discursos oficiales, en sintonía con las leyes fiscales y de copyright.

Sin embargo, y como es de interés de este artículo, al tratarse de cosas que se transan desde el punto de vista del derecho en un escenario "ilícito", éste debiera permitirnos cuestionar las formas que han sedimentado tal representación sobre estos fenómenos, sobre todo en lo que refiere a la íntima vinculación y relación existente entre ley estatal y su falta, avanzando hacia un abordaje que involucre el aspecto social, político, económico y cultural que encierran estas prácticas, e igualmente el modo en que éstas se relacionan con procesos y transformaciones más generales. Es decir, la aspiración de la criminología crítica y los estudios sociales en torno a los comportamientos desviados y su

1 Trabajo inserto en Proyecto FONDECYT 1160963 “Espacialidades transfronterizas en el desierto de Atacama. Movilidad y reconfiguración de identidades nacionales y étnicas".

2 Traducción propia. 
vínculo con las estructuras sociales y sus dinamismos (Becker, 2009; Del Olmo, 1981; Melossi, 2018; Vehlo, 1976).

Esta apuesta analítica y epistemológica pero también política de entender que parte de los fenómenos económicos sancionados bajo un carácter criminal por la ley estatal se hallan vinculados e incluso producidos por el estado (Aguiar, 2015; Heyman; Smart, 1999) -y por ende, su abordaje debiera desarrollarse en conjunto-, comprende que la legitimidad con que el estado reclama un monopolio de la regulación de las formas del comercio, se basa, precisamente, en la deslegitimación de otras, como el robo o la piratería, pues "históricamente, el límite de lo ilícito se ha desplazado de un lado a otro a medida que los bandidos ayudaban a hacer estados y los estados a los bandidos" (Abraham; Van Schendel, 2005, p. 7, traducción propia).

Cuando hablamos de contrabando en nuestra Región, lo estamos haciendo respecto de un grupo de prácticas insertas en lo que se ha nombrado como economías populares (Tassi et al., 2013), particularmente de dinámicas económicas, políticas y culturales que desarrollan sectores marginados y segregados estructuralmente de los dispositivos de integración vía modelos laborales urbanos asalariados tradicionales, aunque vinculadas a éstos. Es decir, de prácticas articuladas en circuitos económicos de todo tipo y que se materializan en un espacio fronterizo y difuso en el que convergen agentes diversos y contingencias, y en el que las tradicionales tipologías relativas a su comprensión, a saber, lícito/ ilícito, formal/informal, acumulación/subsistencia, entre otras, no parecieran ser definitorias ni adecuadas en vista de lo versátiles que suelen ser las disposiciones que ellas adoptan en las espacialidades fronterizas (EF). Por último-y he aquí su especificidad-: son desenvolvimientos laborales inscritos en el aprovechamiento de las asimetrías entre un país y otro, haciendo del cruce de fronteras una actividad económica y política central para poblaciones que habitan EF.

Por tanto, hablamos de desenvolvimientos o "border work" (Reeves, 2014, p. 31) que en su práctica han irradiado mercados de todo tipo con una nueva pléyade de mercancías elaboradas en Asia Oriental, constituyéndose como una forma de trabajo inscrita en la historia de la Región (Laurent, 2014; Rosenblitt, 2013); que goza de una sustantiva legitimidad en las poblaciones locales empero la renovada explotación laboral en que se inscribe (Gago, 2018); y cuya tendencia al alza se ha relacionado con complejos momentos de crisis económica en el mundo (Gago; Cielo; Gachet, 2018, p. 16). 
Estas primeras aproximaciones son muy importantes para comprender el fenómeno del contrabando y su vínculo con las mercancías-imitaciones en la EFAL. Lo anterior, pues tanto la piratería como la reprografía contemporáneas en ésta, y a través de la producción de fakes, alimentan una "formación histórica determinada que ensambla y compone temporalidades, espacios y analíticas críticas de actividades y circuitos de las sociedades contemporáneas" (Gago; Cielo; Gachet, 2018, p. 12), sosteniendo el auge del contrabando en EF, y relacionándose con otros fenómenos, como el alza de los comportamientos laborales cuentapropistas en todo el mundo en el contexto de la contracción y crisis de dispositivos de integración laboral en el neoliberalismo (Sarkar, 2017).

Visto así, el contrabando de mercancías-imitaciones debe ser entendido en un escenario mayor toda vez que se observan, por una parte, modos de articulación de fenómenos económicos globales con "economías comunitarias [...] [dando] cuenta de una nueva combinación de escalas, capaz de ensamblar dinámicas, modos productivos, saberes y circuitos a primera vista incompatibles" (Gago, 2014, p. 102); y por la otra, de procesos que poseen una capacidad de transformación abismante en los patrones de consumo contemporáneos, y que afectan a diversos y amplios grupos sociales (Lin Yi-Chieh, 2011).

La información que aquí se presenta, obtenida a partir del empleo de la etnografía, es parte de una investigación iniciada el 2016 con comerciantes y contrabandistas en la EFAL. El contexto de levantamiento de ésta estuvo marcado por episodios de la conflictiva historia de fronterización de los estados boliviano y chileno: la tensión diplomática entre sus gobiernos debido a los procesos judiciales en curso en la Corte Internacional de Justicia de La Haya (CIJ), en torno a la obligación de Chile de negociar una salida soberana al mar a Bolivia toda vez que éste último país desconoce los alcances de los tratados firmados posteriores a la Guerra del Pacífico -y que la corte falló negando totalmente esta obligatoriedad-; y por la disputa de la propiedad de las aguas relativas al sistema hídrico andino del Silala, que se resolverá durante este año en curso.

Si bien este tipo de situaciones se desarrollan a nivel de los estados centrales, es cierto que imprimen fricción en las poblaciones locales al igual que en agentes reforzadores de fronteras (Grimson, 2011), vale decir, policías y funcionarios que controlan las EF.

Otra circunstancia que tensionó a la EFAL, sucedió el 2017: policías chilenos arrestaron a nueve miembros de la policía y aduana bolivianas, siendo 
encarcelados por casi 100 días en una prisión chilena, acusados, entre otras cosas, de transgresión de frontera, resistencia a la autoridad local e intento de fuga, contrabando y portación de armas cortas y largas.

Considerando lo antes descrito, la aproximación etnográfica empleada, que integra niveles historiográficos y experienciales así como realismo y reflexividad, puso en relación constante la observación y descripción de prácticas con las entrevistas en profundidad y conversaciones realizadas a los diversos actores vinculados al fenómeno a partir de estancias prolongadas en el campo, acción que permitió no circunscribir el método solamente a sus técnicas sino a la construcción de relaciones sociales significativas como parte de una estrategia para distanciarlo de las retóricas que sancionan, por una parte, como criminales las prácticas sociales que realizan estos grupos (Renoldi, 2014, 2015); y por la otra, como un proceso dudoso por la nacionalidad del investigador que lo desarrolla. El trabajo de campo se realizó en momentos de baja densidad en el tránsito en fronteras así como en otros en los que éste aumenta de forma notoria -como sucede en tiempos de festividades y carnavales andinos en la EFAL-y en los que tal situación imprime especificidades sobre esta economía y espacio

En síntesis, y en el marco de su objetivo, el texto que se desarrolla a cuenta de una serie de transformaciones y reordenamientos espaciales en la EFAL, los cuales estarían sosteniendo cambios en las economías y estrategias culturales de la población local, permitiendo que éstas intervengan en la circulación de las cosas que por ahí se movilizan y, simultáneamente, coconstruyendo agencias más flexibles y múltiples en lo que se refiere al consumo de estas mercancías.

\section{Contrabando, mimética y sociedades, o notas para una entrada}

Medina (2001) comprende que los orígenes del contrabando se hallan en la instauración de la propia frontera, sea colonial o moderna. Eminentemente político, su locus sería el de una confrontación "contra el recaudador" (Medina, 2001, p. 479).

Históricamente, el contrabando ha sido observado y representado como una práctica que perjudica al estado desde un punto de vista impositivo. También 
lo haría sobre los grupos humanos y sus ecosistemas, pues esta práctica moviliza cosas que se hallan fuera de norma, como la fitozoosanitaria, regla que si bien fue hegemónica durante la segunda mitad del siglo XX ha sufrido cambios importantes. En efecto, el status que han asumido los delitos de tipo marcario a fines del siglo XX nos habla de novedosos regímenes en el control fronterizo.

El consumo masivo de mercancías-imitaciones en el mundo contemporáneo puede entenderse como un modo de expresión actual de dinámicas sociales constitutivas de la pretensión humana por crear sentidos compartidos y de intimidad cultural, en diferentes escalas témporoespaciales, y en sociedades que se ensamblan en nuevas geografías económicas dinámicas y complejas (Garcés; Maureira, 2018; Morales; Richard; Garcés, 2018; Muñoz, 2016, 2018).

La mimética que encierran estas prácticas, sus impactos en los procesos identitarios y morales de amplios grupos de la población, y en la forma en que estos interactúan y se regulan, se comprende asumiendo al neoliberalismo como la arquitectura material y simbólica de carácter hegemónico en la que éstas se desarrollan. En efecto, esta tecnología de gobierno predominantemente plural e integrativa de otras subjetividades, lógicas o saberes (Gago, 2014), posee especificidades significativas en las formas en torno a las cuales se articuló y articula con los espacios, economías y culturas de la EFAL, desarrollándose de forma desigual y combinada en ésta -como ha observado la geografía al respecto (Santos, 2004; Sassen, 2003).

Visto así, el contrabando se entiende inscrito y como resultado de "una forma sofisticada, novedosa y compleja de enhebrar, de manera [...] íntima e institucional, una serie de tecnologías, procedimientos y afectos que impulsan la iniciativa libre, la autoempresarialidad, la autogestión y, también, la responsabilidad sobre sí" (Gago, 2014, p. 10). Igualmente, alimenta un mercado que se valida e intensifica por promover una cultura de la copia que "emerge como una vía en la que los consumidores pueden resistir y reclamar el control de los significados de un sistema económico cambiante" (Lin Yi-Chieh, 2011, p. 7, traducción propia), con todos los matices que esto pueda implicar.

Remitiéndonos al caso suramericano, la bibliografía advierte la necesidad de comprender las dinámicas relativas al contrabando en tanto situación específica de desenvolvimientos laborales en EF. Su actividad no solo goza de legitimidad social, económica y política en la escala local, en parte por haberse instituido como uno de los mecanismos más importantes del proceso de 
democratización de los mercados ahí existentes y posibilitando un importante proceso de ascenso de grupos sociales tradicionalmente obturados estructuralmente por la colonialidad de los estados suramericanos y el mercado (Müller, 2015; Muñoz, 2018), sino por constituirse como uno de los sectores económicos de mayor importancia en la generación de nuevos empleos para las poblaciones que habitan las EF.

Ejemplo de ello es posible de pesquisar en textos de Cardin $(2013,2014)$ y Cardin y Colognese (2014) relativos a las actividades que cotidianamente realizan sacoleiros, laranjas, cigarreros y bateadores, para dar vida al "mercado informal" en la EF que comparten Argentina, Brasil y Paraguay; o en las prácticas de paseras, en la EF de Encarnación-Posadas (Paraguay y Argentina), y su vinculación con el Estado, la justicia y las diferentes percepciones que se tienen de este comportamiento "desviado" y propio de los "ilegalismos" en los sistemas penales locales (Renoldi, 2008); o en torno a los diferenciales económicos presentes entre los estado de la Región como clave explicativa del comercio ilegal en áreas de frontera (Carrión, 2011); o sobre los culebrones, vale decir, conductores de las actuales caravanas que contrabandean combustible desde Ecuador hacia Perú (Prado, 2012); o de las rutas que las mercancías relativas a la industria del reciclaje de telas supone entre EF y grandes urbes de Brasil (Rabossi, 2008); o de la acción de chamberas o cachineras, y el goteo permanente de juguetes, electrodomésticos y textiles de segunda mano, al igual que papeles higiénicos y ropa deportiva fake, que circulan a diario por la EF de Arica y Tacna (Chile y Perú) (Muñoz, 2016).

Las investigaciones antes señaladas no solo han avanzado en torno a las formas que en la ciencia social existen respecto de cómo pensamos fronteras y espacios, o los regímenes de trabajo, explotación y dinámicas o regulaciones estatales, sino en demostrar el carácter activo, políticamente hablando, que tienen los grupos sociales que ahí habitan, cristalizando una capacidad creativa impresionante para con el aprovechamiento económico, político y espacial de los recursos existentes en las EF a partir del establecimiento de redes y alianzas que, como veremos y a pesar de la contingencia y fricción en la que se inscriben, poseen una fuerza y continuidad central que hemos denominado "gravedad de frontera" (Muñoz, 2016, 2018).

Por último, y si bien los casos que cada una de estas investigaciones aborda poseen características específicas en lo que refiere a aspectos sociales, políticos, 
económicos y culturales, igualmente ello permite observar que las transformaciones y evoluciones de las $\mathrm{EF}$, particularmente las que refieren a las últimas tres o cuatro décadas, se hallan condicionadas y/o determinadas por procesos comunes en diversas escalas, y que bien pueden entenderse dentro de retóricas hegemónicas que sancionan cosas y personas en el neoliberalismo (Merluzzi; Sabatini, 2017), como lo es la lucha contra la ilicitud (Naím, 2006), la aporofobía como discurso sancionador de la movilidad humana contemporánea, específicamente de los grupos sociales empobrecidos (Cortina, 2017), y de la reducción de aranceles para facilitar el tránsito de mercancías e inserciones económicas entre Asia y América Latina (De Miranda; Peláez, 2015).

\section{Entre integración y quiebre, o contrabando y EFAL}

Como parte de una EF trizada por los conflictos bélicos que materializaron los límites de los estados durante los siglos XIX y XX en nuestra Región, los desiertos de Atacama y Lípez, hoy fronterizados por los estados argentino, boliviano y chileno, corresponden por milenios a regiones articuladas espacial, social, económica y culturalmente en Suramérica, nominadas por la arqueología y antropología como "Andes Centro-Sur" (Núñez; Dillehay, 1988; Núñez; Nielsen, 2011) o "Puna de Atacama" (Benedetti; Salizzi, 2011; Morales, 2018; Richard et al., 2018).

Los diferentes grupos sociales que han ocupado esta EF, desarrollaron procesos específicos y dinámicos de habitar los frágiles ecosistemas del desierto, vinculándose tempranamente con los diferentes ciclos económicos que articularon territorios y poblaciones andinas y de forma funcional con cada uno de ellos (Galaz-Mandakovic, 2016). Igualmente, a través de mecanismos y estrategias concretos de relación con los estados coloniales y modernos y los dispositivos de control que ahí fueron instalándose, los que evolucionaron en la medida en que la EF se fronterizaba, construyendo los límites nacionales tal y como los conocemos en la actualidad.

La EFAL, que representa un recorte espacial de esta zona antes caracterizada (ver Figura 1), ha sido objeto de diversas abordajes que analizan las economías que allí tienen lugar, sus formaciones sociales y culturales, los flujos y movilidades que las componen, los regímenes políticos a los que se subordinaron sus espacios y poblaciones, entre otros. 


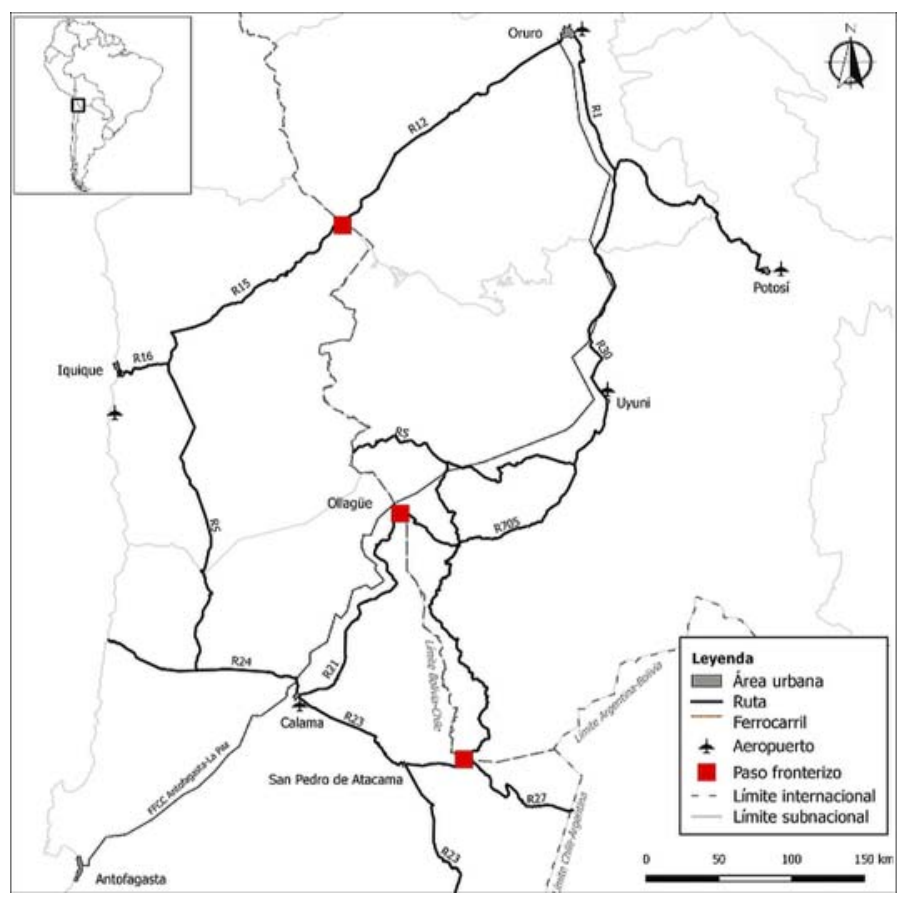

Figura 1. Mapa de la EFAL y redes viales y de transportes más importantes en la actualidad. Los dos controles fronterizos que se encuentran en la parte baja de la imagen, representan, de norte a sur, los complejos fronterizos de Comandante Avaroa y Salar de Ollagüe; y Portezuelo o Hito Cajón y San Pedro de Atacama (Bolivia y Chile). Elaboración propia (2018).

En este sentido, las actuales fronteras nacionales sancionadas jurídicamente a inicios del siglo XX entre Bolivia y Chile, condujeron a la operación de distintos dispositivos o aparatos de nacionalización del territorio de ambos estados, siendo el ferrocarril, dentro de la administración boliviana de la llamada Provincia del Litoral, uno de las más importantes. En efecto, este trazado, inicialmente financiado por la Compañía Huanchaca de Bolivia, permitió articular al puerto de Antofagasta con "Uyuni -y luego Oruro- para exteriorizar la producción de la mina Pulacayo" (Galaz-Mandakovic, 2016, p. 91), la más importante faena argentífera andina pos Potosí. Por el lado chileno y una vez se concretó la ocupación cívico-militar posterior a la Guerra del Pacífico 
(1879-1883), el desarrollo de la industria salitrera y la consecuente "chilenización de Atacama" (González, 2010; Hidalgo, 2004) profundizó aún más este ciclo ferroviario, cimentando así infraestructura vial para vehículos motorizados, y a la que los grupos sociales que circulaban por la EFAL (ahora boliviana y chilena) fueron ajustándose de maneras diversas durante el siglo XX, sobre todo en torno a procesos de formalización e institucionalización de las mismas -y que hasta no hace muchos atrás seguía desarrollándose fuera de los pasos habilitados-. Es interesante observar que el carácter "militar y fiscal" (Medina, 2001, p. 477) que asumen los controles fronterizos en este momento es parte de un mismo proceso de fronterización de estado, cimentando las bases de la movilidad actual.

La EFAL se circunscribe, por el lado boliviano, al Departamento de Potosí, compuesto por 16 provincias de las cuales sólo tres poseen una situación fronteriza con Chile: Daniel Campos, Nor Lípez y Sud Lípez (aunque sólo las dos últimas la tienen con la región chilena de Antofagasta). Por el lado chileno, la EFAL es contenida por la provincia de El Loa -de la Región de Antofagasta-, la única de la región que posee una situación fronteriza con Bolivia.

La administración de los cuatro complejos fronterizos presentes en la EFAL (ver Figura 2) es mixta, es decir, cada país, a una distancia diferida uno del otro (de unos dos a tres kilómetros) se encarga de sancionar y controlar a personas y cosas que por ahí circulan, en términos migratorios, fiscales, aduaneros y fitozoosanitarios. Los controles funcionan en horarios diferidos, pues en Chile lo hacen entre 08:00 y 20:00 horas y en Bolivia lo hacen entre 09:00 y 19:00 horas. Para ambos casos, la red vial tiene características diversas, pues si en el territorio chileno los caminos (Rutas 21 y 23) son asfaltados y/o mejorados con bichosfita hasta prácticamente el límite internacional, en el caso boliviano lo son de tierra y arcilla (Ruta 701 y aledañas).

Es común oír de parte de habitantes de los poblados de la EFAL ante el cruce de la frontera, ideas relativas a aprovechar el viaje "para hacer negocio". La población sostiene que el viaje permite traer quínoa, cereales, hojas de coca o ropa alternativa, "siempre en cantidades pequeñas... ahí usted dice que es

3 El empleo del término "alternativo" se masificó durante la última década para precisamente señalar cosas que no son originales pero que en términos de su estética se acercan a ellos. Hablamos de imitaciones o fakes. 
para consumo suyo, personal"; o bien "películas, discos, pendrives, de esos que vienen de la Zona Franca de Iquique (ZOFRI, Chile) o de Paraguay... de todo puede pasar... Sólo diga que es para usted, que es para uso personal, ahí se ahorra los problemas con los aduaneros". Además de las mercancías antes especificadas, también se alude a tejidos bolivianos que "bien podrían ser vendidos a los extranjeros, a los turista de acá, por el doble o el triple", como puede apreciarse en buena parte de las boutiques de hostales y hoteles existentes en San Pedro de Atacama, Chile.

Respecto de lo anterior, y además del sentido común que manejan las poblaciones de la EFAL que reconoce el potencial y la asimetría entre los países que la componen, es interesante vincular ello a estudios antropológicos que abordan las complejas formas a través de los cuales grupos sociales diversos y en múltiples latitudes del mundo fueron copartícipes del desarrollo de cuadros organizacionales económicos a través de mecanismos de articulación y relacionamiento con el estado colonial y moderno. Si nos situamos desde el período colonial en adelante, podemos observar cómo las poblaciones indígenas van articulando sus lazos políticos y económicos en el marco de las cambiantes lealtades políticas y tributarias que la espacialidad colonial consolidó, y que luego la escala nacional vino a transformar (Mostny, 1954).

De esta manera, el estudio de sistemas económicos que no se rigen del todo por las lógicas del capitalismo o neoliberalismo aunque sí se hallan articulados a éste (Mintz, 1998; Nash, 2008) demostró cómo las relaciones en las que se hallan inmersos diversos tipos de grupos sociales con el estado (o con otro tipo de estructuras políticas) poseían una impronta ambigua y de lealtades aparentemente contradictorias -tanto interna como externamente,simultáneamente adscritas a mecanismos de cooperación temporales y circunstanciales (Aguiar, 2015, p. 542-543).

Estos elementos respecto de la forma en que se articularon estado y grupos sociales bajo ciertos espacios y dinámicas, nos sitúa de forma vertebral en la EFAL en tanto permite aproximarnos a la comprensión en torno a cómo se construye, instala y articula, en una espacialidad como ésta, el habitar propio de las movilidades espaciales vinculadas a la estructuración de una práctica social específica: la del contrabando. 


\section{Cosas y EFAL, o entre aperturas y cierres}

Desde las últimas décadas del siglo XX hasta la actualidad, uno de los impactos más evidentes en la articulación del crisol de economías presentes en las EF de Suramérica con las economías asiáticas y sus vínculos con los puertos australes del Pacífico y Zonas Francas (ZF) ahí existentes, ${ }^{4}$ ha sido la transformación e incremento de las estructuras espaciales y las materialidades asociadas al comercio, en paralelo al sustrato, las prácticas y percepciones de los grupos sociales que ahí lo integran y desbordan.

La aparición de ZF o de regímenes tributarios alternativos en la costa del Pacífico Sur a contar de la segunda mitad del siglo XX y la contracción de iniciativas de integración económica -como el Pacto Andino o el MERCOSUR-en paralelo a la suscripción de tratados de libre comercio de corte neoliberal de fines del siglo XX y principios XXI, han gestado en pocas décadas un intenso escenario de circulación de personas y cosas en la Región. Este escenario posee dinámicas asimétricas, pues si bien la movilidad y libre circulación de personas y cosas no es para nada equivalente en el mundo actual (Velasco, 2016), igualmente ha puesto en evidencia una diversificación de rubros comerciales e itinerarios sociales y laborales en los países donde se observa (Larson; Harris; Tandeter, 1995). Estos procesos se cristalizan en diversas EF, las que comparten como rasgo común la situación de desigualdad estructural respecto de sus centros nacionales, y su histórica articulación como hitos o polos de desarrollo locales y/o regionales dependientes casi exclusivamente de los diferenciales económicos presentes a uno y otro lado del límite internacional (Carrión, 2011).

4 De estas zonas francas de la Región, destaca la ZOFRI, Chile, la más grande e importante de Suramérica a pesar de no ser la más antigua. ZOFRI se encuentra operando desde el año 1975 y es administrada por una entidad privada compuesta por 2.115 empresas que generan alrededor de 36.000 empleos de forma directa e indirecta. ZOFRI establece comercio con más de 70 países, teniendo para 2015 un volumen de comercio de US 2,117 millones. Los dos principales rubros de venta dicen relación con el sector automotriz y los aparatos de electrónica. La presencia asiática es hegemonizada por China, toda vez que su presencia constituye un $17 \%$ del total de las empresas que ahí operan, por una parte, proveyendo el $41 \%$ de total de los productos que ingresan a través de este régimen tributario especial, por la otra. La presencia china se organiza en 67 empresas provenientes principalmente de Shanghai, Ningbo, Hangzhou, Wenzhou, Zhenzhou, Guangzhou, Yiwú y Taiwan, enfocándose en los rubros de las prendas de vestir, el menaje y la electrónica. 


\begin{tabular}{|c|c|c|c|c|}
\hline \multirow{2}{*}{$\begin{array}{l}\text { Complejos } \\
\text { fronterizos } \\
\text { presentes en la } \\
\text { EFAL }\end{array}$} & \multicolumn{4}{|c|}{ Control } \\
\hline & Policial & Migratorio & Fitozoosanitario & Fiscal y tributario \\
\hline $\begin{array}{c}\text { Portezuelo o Hito } \\
\text { Cajón y } \\
\text { Comandante } \\
\text { Avaroa (Bolivia) }\end{array}$ & $\begin{array}{c}\text { Policía Nacional de } \\
\text { Bolivia (PNB), } \\
\text { dependiente del } \\
\text { Ministerio del Interior } \\
\text { del Estado Plurinacional } \\
\text { de Bolivia (EPB) }\end{array}$ & $\begin{array}{c}\text { Dirección General de } \\
\text { Migraciones (DIGEMIG), } \\
\text { dependiente del } \\
\text { Ministerio de Gobierno del } \\
\text { EPB }\end{array}$ & $\begin{array}{l}\text { Servicio Nacional de } \\
\text { Sanidad Agropecuaria } \\
\text { e Inocuidad } \\
\text { Alimentaria } \\
\text { (SENASAG), } \\
\text { dependiente del } \\
\text { Ministerio de } \\
\text { Desarrollo Rural y } \\
\text { Tierras del EPB }\end{array}$ & $\begin{array}{c}\text { Aduana Nacional de } \\
\text { Bolivia (ANB), } \\
\text { dependiente del } \\
\text { Ministerio de Economía y } \\
\text { Finanzas Públicas del EPB }\end{array}$ \\
\hline $\begin{array}{c}\text { San Pedro de } \\
\text { Atacama y Salar de } \\
\text { Ollagüe (Chile) }\end{array}$ & $\begin{array}{c}\text { Policia de } \\
\text { Investigaciones (PDI), } \\
\text { dependiente del } \\
\text { Ministerio del Interior y } \\
\text { Seguridad Pública del } \\
\text { Estado de Chile (EC) }\end{array}$ & $\begin{array}{c}\text { Departamento de } \\
\text { Extranjería y Policía } \\
\text { Internacional de la Policía } \\
\text { de Investigaciones (PDI), } \\
\text { dependiente del } \\
\text { Ministerio del Interior y } \\
\text { Seguridad Pública del EC }\end{array}$ & $\begin{array}{l}\text { Servicio Agricola y } \\
\text { Ganadero (SAG), } \\
\text { dependiente del } \\
\text { Ministerio de } \\
\text { Agricultura del EC }\end{array}$ & $\begin{array}{c}\text { Servicio Nacional de } \\
\text { Aduanas (SNA), } \\
\text { dependiente del } \\
\text { Ministerio de Hacienda } \\
\text { del EC }\end{array}$ \\
\hline
\end{tabular}

Figura 2. Organigrama de las instituciones presentes en complejos fronterizos en la EFAL. Elaboración propia (2018).

En el caso específico de la EFAL, este escenario ha devenido en un aumento y transformación exponenciales de los espacios orientados al comercio y a las ferias -y sus colas ${ }^{5}$-, ocasionando nuevas dinámicas en la lucha contra la ilicitud por parte de los estados boliviano y chileno. Así, a los continuos esfuerzos e intentos de formalización de esta economía provenientes de estos dispositivos, y cuyo propósito ha sido acabar con la exención tributaria y la forma de acceder al espacio público que posee este comercio, hace alrededor de dos décadas se han sumado nuevos crímenes o ilícitos relacionados con mercancías que atentan contra los derechos de propiedad intelectual internacionalmente protegidos, sean imitaciones o "shanzhai" (山寨) (Han, 2016). Este tipo de cosa criminal supone una amenaza real en el territorio de los estados que han suscrito tratados vinculantes en la materia, obligándolos a especializar en cuestiones marcarias a policías y funcionarios de control fronterizo (ver Figura 2 y 3), proceso que no se ha desarrollado homogéneamente entre ambos países.

5 Nombre con el que son reconocidos en Chile las y los comerciantes que aprovechan los espacios comerciales de ferias y mercados para vender productos fuera de tributación ordinaria, por lo general, en el espacio que queda una vez los puestos "formales" terminan. 
Lo anterior, visibiliza la condición circunstancial, dinámica y diversa de las arquitecturas simbólica y material de los marcos normativos y jurídicos con los que se sustenta la acción de los estados, específicamente de sus políticas fiscales y del empleo de la violencia en las EF. Esto, pues si bien hablamos de dinámicas para nada novedosas y propias de las complejas relaciones de fuerza relativas a la fiscalidad y a la aduana como elementos determinantes en la instauración de un tipo de frontera, también lo hacemos respecto de la tendencia global en la que el control fronterizo contemporáneo de personas y cosas se sustenta en un aparato burocrático, militar y de monetarización creciente (Medina, 2001), y en el que la biotecnologización en sus prácticas de control ha alcanzado una importancia central (Machado, 1998, 2005).

En la última década, Bolivia y Chile han experimentado un crecimiento significativo de las demandas por infracción marcaria, sea piratería o reprografía. Tanto el Servicio Nacional de Propiedad Intelectual (SENAPI), creado en 1997 por el estado boliviano, así como las policías, el Servicio de Impuestos Internos (SII)

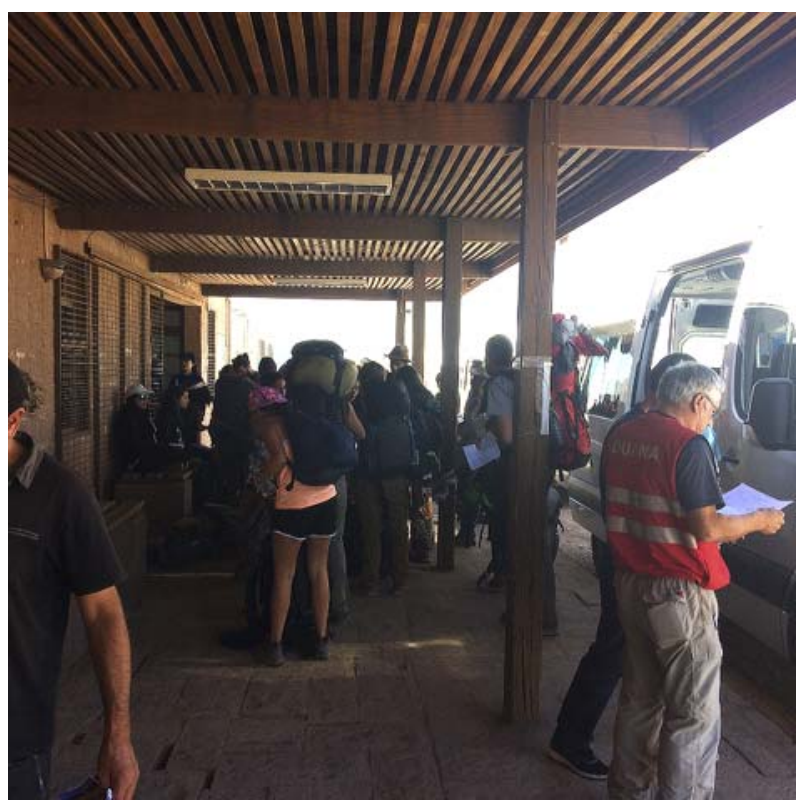

Figura 3. En el control fronterizo de San Pedro de Atacama, funcionarios del SNA inspecciona vehículos que acaban de regresar de un tour desde Uyuni, Bolivia, justo antes de pasar al control en sala. Según cifras de esta institución, la Región de Antofagasta presenta la mayor tasa de infracción por contrabando de piezas de interés arqueológico a nivel nacional, seguido por la infracción de carácter marcario. Fuente propia (2018). 
e incluso el Ministerio Público chilenos, amparados por la Ley no. 17.336 sobre Propiedad intelectual y Derechos de autor del año 1970, persiguen y sancionan este práctica, cuyo aumento bordea un $150 \%$ por año desde comienzos de este milenio. Por lo mismo, es común escuchar en la EFAL, y de parte de comerciantes vinculados a rubros tradicionales como la venta de hierbas o tejidos, que las mercancías-imitaciones -sobre todo provenientes de China- "están invadiendo el comercio" bajo el rótulo de alternativas. Este comentario comúnmente se asocia a la dudosa calidad de tales productos y lo desechable que terminan siendo éstos.

El impacto de estas cosas ha permeado aún más en países -o áreas específicas de éstos, como lo es el caso del sur de Bolivia- que no poseen empresas transnacionales de retail y donde la venta al detalle se diversifica en pequeños comercios familiares que se proveen a través de cadenas articuladas y solidarias con el contrabando, abasteciéndose de mercancías elaboradas en países en los que las regulaciones de copyright son de mínima intensidad o bien inexistentes.

\section{Cruzar, volver a cruzar, o la vida del contrabando en la EFAL}

El contrabando en la EFAL es un tipo de actividad que se enfrenta hasta el día de hoy con agencias tributarias y policiales por su práctica, aunque simultáneamente en este proceso ha adquirido un valor local elemental, fundamentalmente por ser el proveedor de artículos electrónicos de confianza y a bajos precios, por una parte; y por la otra, por ser capaz de coconstruir, transformar y aumentar los espacios comerciales en los que se ha venido desarrollando y, así, incorporar a importantes sectores de la población de las EF al mundo laboral.

Según el diario financiero latinoamericano América Economía (Bolivia..., 2012), el 50\% de las mercancías que ingresan a la ZOFRI tienen como destino el territorio boliviano. Esta cifra, que se condice con los registros web de la ZF, permite observar que hoy por hoy es la puerta de conexión del Estado Plurinacional de Bolivia -y ciertamente también del Norte Grande de Chile y de otras zonas de la Región-con los mercados de Asia Oriental, específicamente chinos.

A pesar de la regulaciones y controles fronterizos tanto bolivianos como chilenos, y de la política de tolerancia cero con la actividad criminal del contrabando por parte de los estados -y cuyos discursos se centran paradójicamente en el narcotráfico y la trata de personas-, éste se desarrolla en una tendencia al alza en 
la EFAL, sobre todo el caso de la ropa fake (ver Figura 4) o bien de las mercancías electrónicas chinas, las que en ambos países sustentan la existencia de más de 1000 ferias dedicadas a su venta (En Bolivia..., 2018). Por lo mismo, tal y como observa Dayana, funcionaria del SNA en el control fronterizo Salar de Ollagüe,

Tienes a mucha gente tratando de hacer negocios con los productos que obtienen acá [Chile] a un precio muy accesible, porque [...] está la ZOFRI, Calama, y todo lo que hay por acá sabemos que viene de allá o de Arica, que también es muy barato. Y esas personas ocupan las fechas de las ferias para pasar todo lo que puedan para vender allí, supuestamente, porque nosotros sabemos que no es así. Ponte: una vez quería pasar un comerciante de Calama, que es chileno pero que tiene familia en Uyuni o en Alota, no sé bien dónde... y venía con un camión lleno, lleno de lavadoras, de esas antiguas, que no son automáticas. Que son como un tambor, que no centrifugan [...] y se suponía que las llevaba a la feria, pero ¿para qué quiere pasar con 60 u 80 lavadoras de ese tipo? Na' que ver porque por ese tipo de conductas, otros que sí están llevando cosas para la feria del trueque, nos alegan que porque los revisamos tanto y qué sé yo [...] Entonces, claro, lo mismo pasa con colchones, con microondas... hasta con herramientas, martillos, alicates.

Del texto anterior, es posible observar cómo esta funcionaria chilena entiende las ventajas comparativas en la adquisición de productos en Chile para con su venta en Bolivia, identificando a la ZOFRI como un elemento central en la circulación de mercancías en la EFAL a través del contrabando. Además de ello, señala a la "Feria del trueque o binacional" ${ }^{6}$ como una instancia importante empleada por comerciantes de la EFAL para la realización del contrabando de productos que se eximen de las tasas de gravamen fiscales y de la inspección por delitos marcarios.

6 En cualquiera de los dos casos, la funcionaria se refiere a una instancia comercial que se realiza en la frontera entre Bolivia y Chile, justo por delante de las localidades de Avaroa y Ollagüe y sus complejos fronterizos (Comandante Avaroa y Salar de Ollagüe). Propia de una tradición de intercambio de las poblaciones quechua y aymara locales, este momento de intercambio posee más de quince años de historia, y viene a formalizar el intercambio de diversos productos, los que mayoritariamente eran de origen silvoagropecuario en sus inicios a través del trueque, a pesar de existir igualmente intercambios en dinero. En la actualidad, los productos que hegemonizan este intercambio, el que se basa exclusivamente en compra y venta a través de moneda boliviana y chilena, dólares o euros, son los electrónicos, ya sean originales o "alternativos" (mercancías-imitaciones), y ropa para climas extremos, proveniente de mineras transnacionales aledañas a la zona (San Cristóbal en Bolivia, y Collahuasi en Chile). 


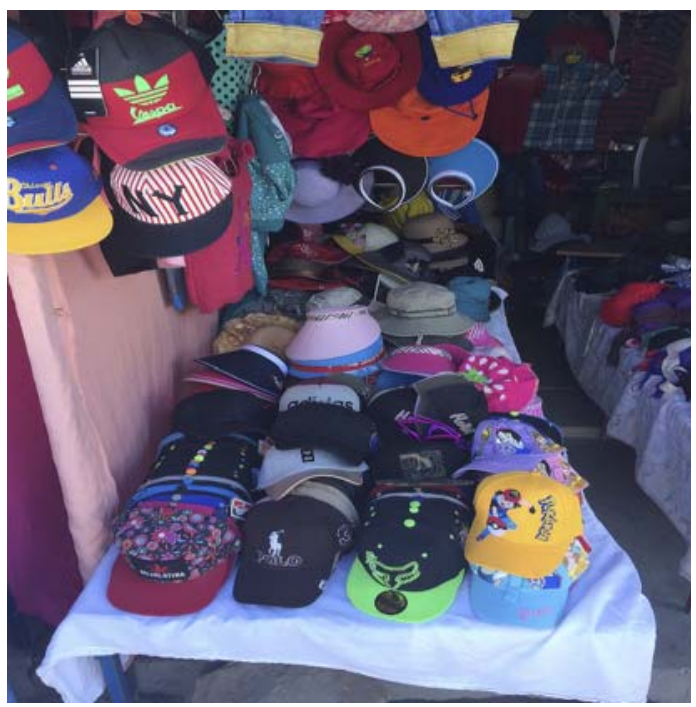

Figura 4. Vista de las mercancías que ofrece tienda del Mercado Antofagasta, en el centro de Uyuni. Es común que el comercio que antes se orientaba a la venta de textiles andinos haya comenzado a hacerlo a la venta de mercancías-imitaciones, en este caso ropa fake. Según las y los comerciantes locales que se proveen de estos productos desde La Paz, Bolivia, o Calama, Chile, tanto esta pléyade de mercancías como la relativa a la telefonía móvil y electrónica, es la más rentable en estos momentos, desplazando a los oficios tradicionales de estos espacios. Fuente propia (2018).

A menos de 200 kilómetros del control fronterizo chileno en Ollagüe, se encuentra Uyuni, ciudad boliviana en la que el joven matrimonio de Vlado y Freda se dedica a la venta de electrodomésticos, artículos electrónicos y vehículos menores chinos (Figura 5). Dentro de sus estrategias de venta, el empleo del término "alternativo" -con el fin de no aludir a la procedencia mayoritariamente china de sus productos-, es central. Según ellos, el mercado local aún resiente la procedencia de los electrodomésticos chinos, en particular por poseer una suerte de estigma en términos de calidad y durabilidad. Sin embargo, la mercancía se ha instalado y se vende con rapidez, porque tal estigma no es del todo cierto, y porque las mercancías-imitaciones son las únicas existentes en ciertos géneros (ver Figura 6). El segmento joven de la población desconoce otros productos que no sean los que se ofertan en la actualidad, por lo que consumen -según estos comerciantes- dos o tres teléfonos móviles por año.

Este reacomodo de símbolos que caracteriza el matrimonio de comerciantes, nos habla de transformaciones profundas en los niveles organizacional e institucional, toda vez que las agencias de la población en la EFAL han venido dando lugar a percepciones más flexibles y múltiples en lo que se refiere al consumo de estas mercancías, fundado sobre nuevas experiencias sobre productos fakes y su calidad. 
Expresión de los grupos sociales ascendentes a través del comercio de productos que ingresan por contrabando a Bolivia, es común que los comerciantes uyunenses que se dedican a su venta hayan dejado atrás el trabajo agrícola en las inmediaciones de Uyuni o Oruro para así instalarse en el espacio comercial de la ciudad. Al hacerlo cerca de la Terminal de Buses, una parte de las tiendas se emplea como bodega para mercancías que están de paso, buena parte de tipo fake, y en dirección a otras ciudades bolivianas, como Tupiza y Villazón (ciudades también fronterizas, en este caso con el norte de Argentina), o bien hacia el norte de Argentina.

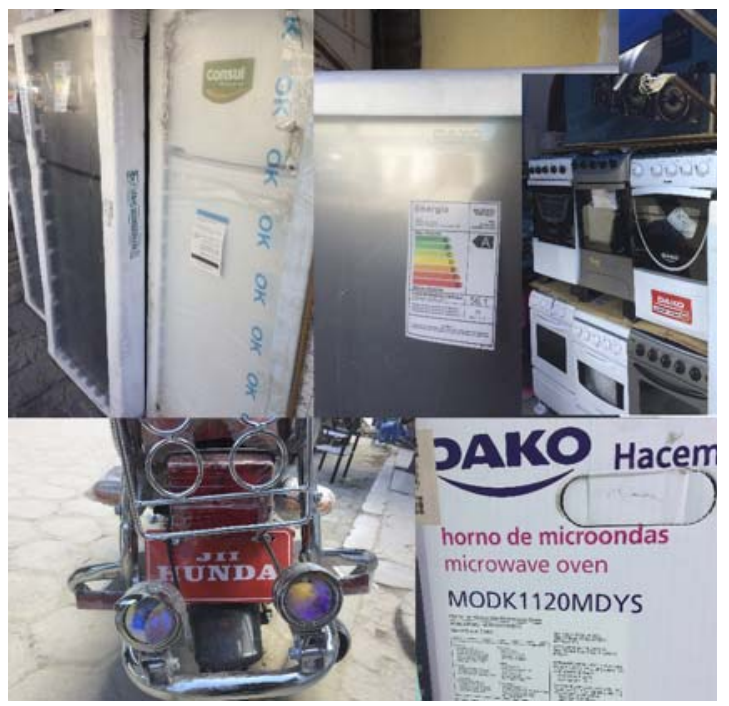

Figura 5. Artículos electrónicos originales y "alternativos" (fakes) ofertados en el centro de la ciudad de Uyuni. Nótese la motocicleta marca "Hunda". Elaboración propia (2018).

\section{Transformación y ensamblajes, o la complejidad de lo formal/ informal, lícito/ilícito}

Sentado en la butaca del conductor, Marco, comerciante de Uyuni dedicado a prestar servicios de trasporte a mineras locales y a "mover mercancías con facturas desde Avaroa", conversa exaltado con uno de sus empleados a través de su móvil Huawei. Lo que acaba de suceder es grave: han requisado 12 cocinas y una cantidad similar de freezeres por haber superado el límite de la "menor 
cuantía". A Ahora, para recuperar estas mercancías, sólo queda pagar una internación ya amonestada por la ANB, agencia que comprobará antes que no se trata de un delito de contrabando. De ser así, la multa será muy alta.

El pedido retenido es esperado hace días por otra comerciante de la ciudad, con nacionalidad boliviana pero que sólo vende "productos chilenos", quien debe despacharlo a sus compradores que accedieron a ellos pagando por adelantado. Su ex esposo es quien adquirió los productos, pues posee nacionalidad chilena hace una década. Él se dedica al rubro de transportes en la megaminería de Calama, y espera su parte del pago, la que debe costear el pago de los electrodomésticos que han sido comprados en tres cuotas en una famosa casa del retail local.

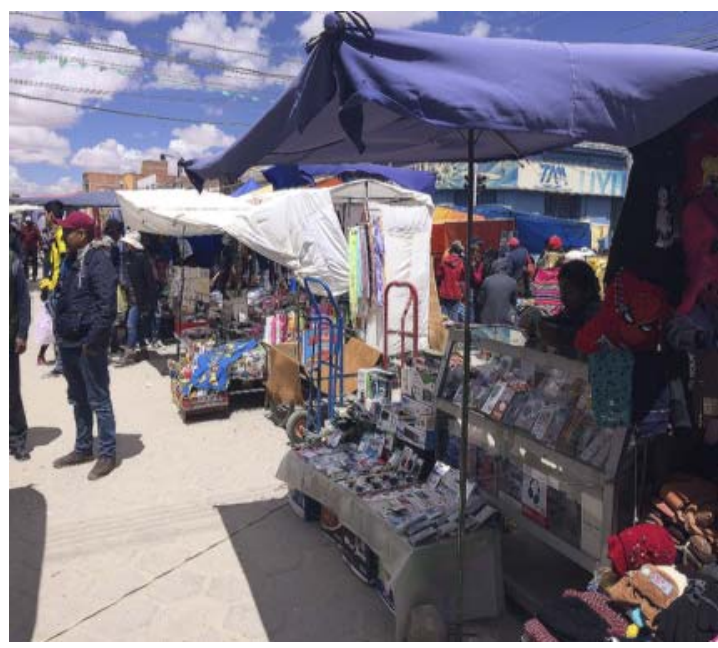

Figura 6. Puesto de mercancías-imitaciones en la Feria de Uyuni. Es común que en éstos espacios se comercien productos "alternativos" de marcas como "Samsong", "Sonya" o "Panasonico". Uno de los ámbitos de control que mayormente se ha robustecido a partir del uso de escáneres o tecnología radioscópica de control no invasivo en la EFAL ha sido el relativo al marcario. Lo anterior, se conecta con la instalación a fines de 2017 del primer escáner para control fronterizo en Comandante Avaroa -aparato que existe desde mediados de los años ‘90 en Salar de Ollaguie, del lado chileno-. Tal situación ha permitido aumentar considerablemente los impuestos a las mercancías chinas provenientes de la ZOFRI, Chile, sean originales o no; y por la otra, articularse como una nueva impronta o discurso de control fronterizo nacional tendiente a mostrar que el comercio en Bolivia no es solidario con la piratería global, entregando, con esto, mayor confianza a mercados internacionales, como el europeo o norteamericano, según la ANB. Fuente propia, 2018.

7 Menor o Mínima Cuantía es un régimen de importación de bienes en vigencia desde fines de 2016. Promovido por la ANB, pretende intervenir en el contrabando de mercancías sancionando los ingresos máximos de mercancías que no pagan los costos de nacionalización (por un valor no superior a los USD 2000 y cada 90 días). De superar el valor o tiempo, la ANB puede cobrar un $21 \%$ del valor de la mercancía y una amonestación de hasta el 50\% del valor bruto de ésta. 
Marco transita por la ciudad en su Toyota Hilux del año. "El negocio ha estado bueno", insiste en repetir cada vez que lo veo, por lo general bebiendo alguna bebida gaseosa. De color blanco, el tono de su vehículo destaca por el barro existente en rutas locales. Hace menos de cinco años, decidió apostar por el traslado de los trabajadores que requieren las faenas de la una minera del Departamento, poniendo a disposición de su gerencia la flota de tres buses y una serie de horarios que le acomodaban a los trabajadores que ingresaban o salían de sus labores. Ahí logró consolidarse como el transporte oficial de ésta, para luego comenzar a tejer redes con otros comerciantes de la EFAL, y enlazarse hacia la costa antofagastina. Sin sus primos (políticos y no consanguíneos), con los que no tenía contacto desde que salió de La Paz durante la década de los ' 80 , este proceso no hubiera sido tan exitoso. Uno de ellos, por ejemplo, vivió por dos años en la ciudad de Guangzhou, China, para facilitar el negocio de traer productos de mejor calidad desde allá, evitando la ZOFRI.

Hace poco más de una década, Marco se dedicaba a picotear ${ }^{8}$ diversos productos electrónicos, fundamentalmente electrodomésticos de segunda mano desde Calama. Para ser picotero, sólo se necesitaba tener una camioneta, "perder miedo", y una red de familia y amigos, comenta. Ahí movía decenas de lavadoras y microondas; también freezeres.

Sin embargo, actualmente Marco tributa. Su opinión, generalizada entre comerciantes que poseen régimen simplificado o general en Bolivia, se acompaña de un discurso a favor del gobierno masista de Morales y las articulaciones que éste ha generado con China, tendiendo a subrayar el hecho de que el país necesita de recursos fiscales para implementar cambios y políticas públicas, los que por otra vía serían muy difíciles de obtener.

Este imaginario tributario se desarrolla en momentos en que la política fiscal de los estados boliviano y chileno no logra consolidarse hegemónicamente en la EFAL y en el que otros recursos o elementos, como lo son las redes étnicas o comerciales fronterizas, asumen un protagonismo antes inexistente.

Las tensiones actuales entre Bolivia y Chile han repercutido en un mayor control en las fronteras por parte de los agentes de estado relativos a éste (ver Figura 7), generando, primero, procesos de reinvención en las formas de contrabandear que

Contrabandear por rutas no habilitadas e ilegales. 
emplean los comerciantes locales de la EFAL (como el caso de Marco lo ejemplifica); y segundo, modificaciones en las redes de aprovisionamiento.

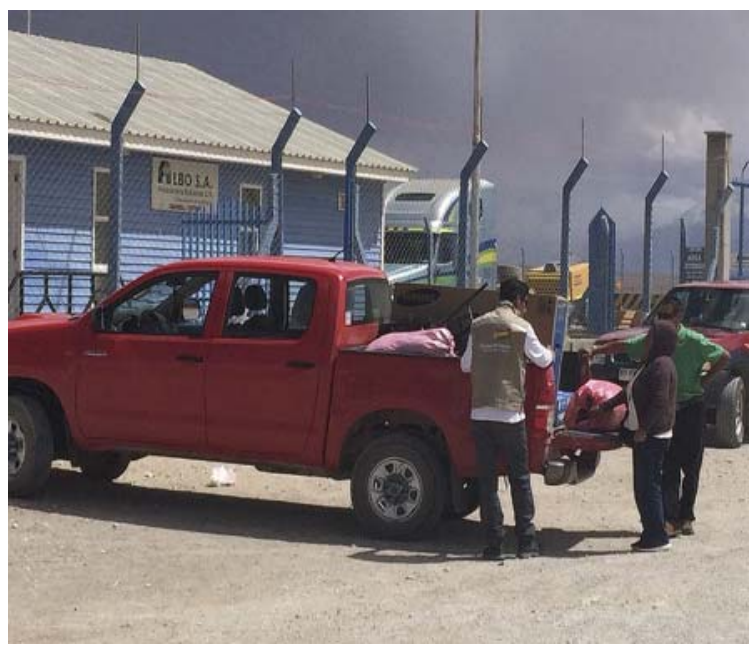

Figura 7. Funcionario de la ANB requisa mercancías, específicamente ropa fake, en el control fronterizo Avaroa, Bolivia. Estos agentes, y como parte de una estrategia para evitar contubernios entre población y Servicio, cuestión que sucede con frecuencia -sostienen-, son cambiados de control permanentemente. Fuente propia (2018).

Al respecto del primero, y si bien era tradicional contar dentro de la red de aprovisionamiento con alguna persona "con carnet de Chile" y residencia en tal territorio debido a las ventajas que significaban los precios de los artículos (imitaciones o no) electrónicos en el retail local, el mayor control en las fronteras ha obligado a reducir la intensidad de ciertos productos ${ }^{9}$ en los últimos años. Al respecto, María, mujer boliviana de Uyuni, de 34 años de edad, y trabajadora de una reconocida empresa de transporte de pasajeros y encomiendas de la EFAL, comenta que:

Los primeros años, se veía mucho el transporte de cosas eléctricas, lácteos, había mayor frecuencia de cosas. Pero este año, del 100\% ha bajado un $80 \%$... Yo le digo, porque antes más que pasajeros había mercaderías. Pero en este tiempo ya no se

9 En fechas cercanas a carnavales, muchos comerciantes indican que el control se reduce, específicamente en torno a productos que se emplean dentro de tales festividades, como lo son las bebidas alcohólicas, textiles tradicionales y frascos de nieve artificial. 
puede: del lado chileno, todo es con factura, entonces no puedes traer tres lavadoras, o cuatro lavadoras... Ya nada en cantidad, sólo uno, y con factura. Y tienes que esperar tres meses para volver a traer uno [...] Antes esto generaba dinero para todos, chilenos y bolivianos... Imagínese usted que antes veinte comerciantes bolivianos de Uyuni iban a Falabella o ABCDin, y compraban ropa, traían cosas eléctricas... Pero ya no se puede, entonces ha disminuido también la cantidad de comerciantes [...] En la actualidad, entonces, para ellos [los comerciantes] es más fácil traer del Brasil, del Perú... Y porque desde allá es más fácil y simple pagar... no están estricto como en Chile. Entonces, esta gente está trayendo cosas eléctricas del Perú, están trayendo ropa del Perú, de Desagüadero... Y también desde la Argentina, de por Villazón, aunque quede un poquitito más lejos, es más accesible.

Respecto del segundo punto, vale decir, las modificaciones en las redes de aprovisionamiento, resulta importante subrayar, como observa María, el hecho de que si bien las mercancías (sean imitaciones o no) pueden provenir de otros países o rutas, éstas siguen siendo elaboradas e importadas desde China o, como insiste Wilfredo, joven comerciante uyunense: "el producto es el mismo, hermano: si lo único que cambia es dónde y quién lo arma”. Así, el sector de transporte de personas y cosas se ha ido rearticulando de manera solidaria con el comercio local, vinculándose con nuevas EF de la Región, como lo es el caso de Puno, Perú, o Corumbá, Brasil. En estas EF el trabajo de los contrabandistas suele ser más sencillo, aunque igualmente controlado.

\section{El espacio y la economía del contrabando en la EFAL}

En la última década, entre las Avenidas Potosí y Cabrera, en el centro de Uyuni, las tiendas orientadas a satisfacer la elevada demanda de electrodomésticos y artículos electrónicos local ha crecido de manera sostenida. Este fenómeno es igualmente observable en Calama u Ollagüe, aunque ahí ha sido el retail el que lo ha absorbido.

Empero, hace algunos años en Uyuni, el espacio comercial céntrico era utilizado para carnicerías o abarroterías, rubros se han ido desplazando hacia las periferias con el pasar de los años. Las viejas casonas de adobe han sido 
refaccionadas para ser empleadas como galerías comerciales, con varias tiendas en su interior; y las menos, aunque importantes, sobre todo en el centro de la ciudad, se han convertido en edificios estilo cholet, montándose galerías en vertical en las que también conviven hostales y gimnasios (ver Figura 8).

Actualmente, los comerciantes vinculados a la venta de productos electrónicos, en su amplia gama de variedades, están financiando obras de renovación urbana en la ciudad, aunque no de uso público sino orientadas a acrecentar su presencia por medio de la ampliación del espacio comercial disponible, proceso que segrega a comerciantes que no puede costear los altos costos de alquiler. Este proceso de transformación de las estructuras espaciales se vincula con el aumento y crecimiento de las ferias, toda vez que en ellas se ha ido integrando la población segregada y no absorbida por este proceso ni los mercados más tradicionales.

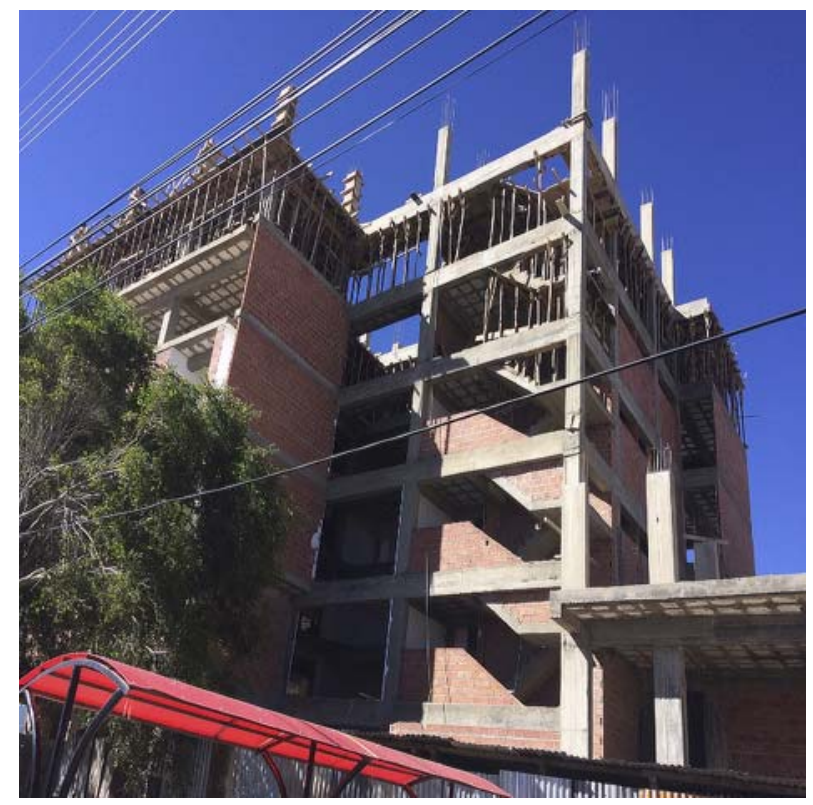

Figura 8. Construcción de galería comercial vertical en el centro de Uyuni. Buena parte de la renovación arquitectónica de la ciudad está siendo realizada a través de inmuebles orientados a ampliar el espacio comercial disponible, debido, entre otros fenómenos, al intenso público que articula el etnoturismo en la EFAL. Fuente propia (2018). 


\section{Consideraciones finales}

Los momentos de ajuste y despojo macroeconómicos en el mundo relativos a las crisis económicas estructurales de las décadas de 1970, 1980 y 1990, y las más recientes, se constituyeron como una matriz elemental para la masificación de actividades propias de las economías populares en nuestra Región, sacudiendo y diversificándose en amplios grupos sociales. Estos momentos de ajuste no fueron otra cosa que los modos en que el neoliberalismo y su proyecto civilizatorio se espacializó en el mundo, y que en el contexto suramericano se viabilizó a través de las dictaduras militares que azotaban la Región (Lander, 2000, p. 246). Y como tal modelo combina desigualdades y asimetrías de forma multiescalar, produjo un espacio y condiciones para que el que el comercio y contrabando en la EFAL se viera reforzado; y ante la crisis de los mercados laborales urbanos, se intensificara.

Así, "más allá del contrabando y de lo ilícito, millares y hasta millones de sujetos construyeron sus mundos en un universo marcado por la acomodación a la diversidad cultural" (Pinheiro-Machado, 2010, p. 117), a la que incluso los estados les otorgan una ambigua legitimidad a través de políticas informales (Cross, 1998; Müller, 2015).

Los procesos de circulación en la EFAL vinculan a agentes comerciales presentes y no presentes en tal espacialidad, provenientes tanto de países y regiones diferenciadas así como de sectores económicos formales/informales, legales e ilegales, entendiendo que tales categorías políticas han sido definidas y reguladas históricamente por las élites (Ribeiro, 2007, p. 42). Estas articulaciones, en fricción permanente tanto por las características propias del comercio como también por las políticas de control fronterizo a uno y otro lado del límite internacional, suponen complejos equilibrios y cambios sistemáticos en las estrategias empleadas con el comercio de estas cosas, afectando otras instituciones sociales, como lo es el caso del parentesco y las redes familiares y laborales, o bien la contigüidad y relación con otras EF.

El impacto de estos reordenamientos políticos y económicos en la EFAL, está produciendo dinámicas en las que las mercancías-imitaciones vienen a producir unos espacios que ven transformadas sus históricas dinámicas de relaciones sociales y de intercambio. Si la etnohistoria poseía como clave hegemónica la organización del mundo andino a través de la complementariedad 
de pisos ecológicos puna-costa (Murra, 1975), la introducción de nuevas mercancías en la EFAL viene a articular una nueva geografía económica en la que los intercambios históricos o tradicionales se relacionan y subordinan a esta nueva pléyade de mercancías, la que permite a su vez nuevas formas de empresarialidad y articulación fronterizas (Garcés et al., 2018).

A pesar del estigma del contrabando, la ciencia social debe avanzar hacia estudios que aborden la forma en que este tipo de práctica comercial se enlaza con otras, al punto de estar participando de transformaciones radicales en torno al espacio y la materialidad comercial y urbana. Este poder no sólo renueva las ciudades en las EF sino que las está transformando funcionalmente para con la circulación y almacenaje de personas y cosas, orientando y adecuando materialidades para ello. Vemos así la capacidad performativa y determinante que posee este fenómeno en los modos de vida locales (Cardin, 2014) al igual que lo activo que se encuentran en éste los procesos de diferenciación, desigualdad y combinación posibles en torno a la interacción entre dinámicas locales y multiescalares.

\section{Referencias}

ABRAHAM, I.; VAN SCHENDEL, W. Introduction. The making of illicitness. In: VAN SCHENDEL, W.; ABRAHAM, I. (ed.). Illicit flows and criminal things: states, borders, and the other side of globalization. Bloomington: Indiana University Press, 2005. p. 1-37.

AGUIAR, J. Estados de simulación: piratería, contrabando, neoliberalismo y el control de la ilegalidad en América Latina. In: ALBA, C.; LABAZÉE, P. (ed.). Metropolización, transformaciones mercantiles y gobernanza en los países emergentes. Ciudad de México: Colegio de México, 2015. p. 541-592.

BECKER, H. Outsiders: hacia una sociología de la desviación. Buenos Aires: Siglo XXI, 2009.

BENEDETTI, A.; SALIZZI, E. Llegar, pasar, regresar a la frontera. Aproximación al sistema de movilidad argentino-boliviano. Transporte y Territorio, Buenos Aires, n. 4, p. 148-179, 2011.

BOLIVIA importa mercadería de Iquique por más de US\$ 900 millones. América Economía, 1 abr. 2012. Disponible en: https://www.americaeconomia.com/ economia-mercados/comercio/bolivia-importa-mercaderia-de-iquique-pormas-de-us900-millones. Acceso: 10 dic. 2018. 
CARDIN, E. La historia de una vida en situación de frontera: migración, superación y trabajo en el "circuito sacoleiro". Revista de Estudios Sociales, Bogotá, n. 48, p. 100109, 2013.

CARDIN, E. Teoria das fronteiras e totalidades. In: CARDIN, E.; COLOGNESE, S. (org.). As ciências sociais nas fronteiras: teorias e metodologias de pesquisa. Cascavel: JB, 2014. p. 43-59.

CARDIN, E.; COLOGNESE, S. (org.). As ciências sociais nas fronteiras: teorias e metodologias de pesquisa. Cascavel: JB, 2014.

CARRIÓN, F. Economía de frontera: una atracción fatal. Fronteras, Quito, n. 7, p. 1, 2011. CORTINA, A. Aporofobia, el rechazo al pobre: un desafío para la democracia. Madrid: Paidós, 2017.

CROSS, J. Informal politics: street vendors and the state in Mexico City. Stanford: Stanford University Press, 1998.

DE MIRANDA, M.; PELÁEZ, J. (comp.). Las relaciones económicas entre América Latina y Asia: hacia una construcción de una nueva inserción internacional. Cali: Sello Editorial Javeriano, 2015.

DEL OLMO, R. América Latina y su criminología. Ciudad de México: Siglo XXI, 1981.

EN BOLIVIA hay 1.031 ferias de ropa usada. El Día, 3 abr. 2018. Disponible en: https:// www.eldia.com.bo/index.php?cat=357\&pla=3\&id_articulo=248201. Acceso: 10 dic. 2018.

GAGO, V. La razón neoliberal: economías barrocas y pragmática popular. Buenos Aires: Tinta Limón, 2014.

GAGO, V. What are popular economies? Some reflections from Argentina. Radical Philosophy, London, year 2, n. 2, June 2018. Disponible en: https://www.radicalphilosophy.com/article/what-are-popular-economies. Acceso: 5 dic. 2018.

GAGO, V.; CIELO, C.; GACHET, F. Economía popular: entre la informalidad y la reproducción ampliada. Íconos, Quito, n. 62, p. 11-20, 2018.

GALAZ-MANDAKOVIC, D. Inclusions, transformations et asymetries du capitalisme minier sur la cote d'Atacama: les dérives de la production thermoelectrique a Tocopilla (Chili) 1914-2015. 2016. Tesis (Doctorado en Historia y Antropología) - Instituto de Arqueología y Antropología, Universidad Católica del Norte, San Pedro de Atacama; Université Rennes 2, Rennes, 2016.

GARCÉS, A.; MAUREIRA, M. De familia a organización étnica: redes para una espacialidad transfronteriza en la Puna de Atacama. Revista Chilena de Antropología, Santiago, n. 37, p. 230-248, 2018. 
GARCÉS, A. et al. Formas porosas. Tiempos, movilidad y economías de frontera entre San Pedro de Atacama y Lípez. Revista de Dialectología y Tradiciones Populares, v. 73, n. 2, p. 547-568, 2018.

GONZÁLEZ, J. La provincia de Antofagasta. Creación y consolidación de un territorio nuevo en el Estado chileno: 1888-1933. Revista de Indias, v. 70, n. 249, p. 345-380, 2010.

GRIMSON, A. Los límites de la cultura: crítica de las teorías de la identidad. Buenos Aires: Siglo XXI, 2011.

HAN, B. Shanzai: el arte de la falsificación y la deconstrucción en China. Buenos Aires: Caja Negra, 2016.

HEYMAN, J; SMART, A. Status and illegal practices: an overwiew. In: HEYMAN, J. (ed.). States and illegal practices. Oxford: Berg, 1999. p. 1-24.

HIDALGO, J. Historia andina en Chile. Santiago: Editorial Universitaria, 2004.

LANDER, E. La colonialidad del saber: eurocentrismo y ciencias sociales: perspectivas latinoamericanas. Buenos Aires: CLACSO, 2000.

LARSON, B.; HARRIS, O.; TANDETER, E. (ed.). Ethnicity, markets, and migration in the Andes: at the crossroads of history and anthropology. Durham: Duke University Press, 1995.

LAURENT, M. Contrabando, poder y color en los albores de la República: Nueva Granada 1822-1824. Bogotá: UNIANDES, 2014.

LIN YI-CHIEH, J. Fake stuff: China and the rise of counterfeit goods. London: Routledge, 2011.

MACHADO, L. Limites, fronteiras, redes. In: STROHAECKER, T. et al. (org.). Fronteiras e espaço global. Porto Alegre: AGB, 1998. p. 41-49.

MACHADO, L. Ciência, tecnologia e desenvolvimento regional na Faixa de Fronteira do Brasil. Parcerias Estratégicas, Brasília, n. 20, p. 747-765, 2005.

MEDINA, E. Contrabando en la frontera de Portugal: orígenes, estructuras, conflicto y cambio social. 2001. Tesis (Doctorado en Antropología) - Universidad Complutense de Madrid, Madrid, 2001.

MELOSSI, D. Controlar el delito, controlar la sociedad: teorías y debates sobre la cuestión criminal, del siglo XVIII a XXI. Buenos Aires: Siglo XXI, 2018.

MERLUZZI, M.; SABATINI, G. Introducción. In: FAVARÒ, V.; MERLUZZI, M.; SABATINI, G. (ed.). Fronteras: procesos y prácticas de integración y conflictos entre Europa y América (siglos XVI-XX). Madrid: Fondo de Cultura Económica, 2017. p. 11-24. 
MINTZ, S. Dulzura y poder: el lugar del azúcar en la historia moderna. Ciudad de México: Siglo XXI, 1998.

MORALES, H. Habitar el desierto: cuadernos de campo de la Puna atacameña. Santiago: SNPC, 2018.

MORALES, H.; RICHARD, N.; GARCÉS, A. Capitalismo en el desierto. Materialidades, espacios y movimiento. Revista Chilena de Antropología, Santiago, n. 37, p. 76-82, 2018.

MOSTNY, G. Peine: un pueblo atacameño. Santiago: Instituto de Geografía, Universidad de Chile, 1954.

MÜLLER, J. Etnografía del área comercial Eloy Salmón (La Paz, Bolivia): transformaciones territoriales, estrategias económicas y prácticas culturales. Temas Sociales, La Paz, n. 37, p. 13-34, 2015.

MUÑOZ, J. De chamba en chamba, o el espacio y la economía del contrabando en el espacio fronterizo Arica-Tacna. 2016. Tesis (Maestría en Antropología) - Instituto de Arqueología y Antropología, Universidad Católica del Norte, San Pedro de Atacama, 2016.

MUÑOZ, J. Trailers, comercio y picoteros. Aproximaciones al espacio del contrabando en la espacialidad fronteriza Atacama-Lípez. Revista Chilena de Antropología, Santiago, n. 37, p. 267-286, 2018.

MURRA, J. Formaciones económicas y políticas del mundo andino. Lima: Instituto de Estudios Peruanos, 1975.

NAÍM, M. Ilícito: cómo traficantes, contrabandistas y piratas están cambiando el mundo. Buenos Aires: Sudamericana, 2006.

NASH, J. Comemos de las minas y las minas nos comen a nosotros: dependencia y explotación en las minas de estaño bolivianas. Buenos Aires: Antropofagia, 2008.

NÚÑEZ, L.; DILLEHAY, T. Camelids, caravans, and complex societies in the southcentral Andes. In: SAUNDERNS, N.; MONTMOLLIN, O. (ed.). Recents studies in preColumbian archaelogy. Oxford: BAR IS, 1988. p. 603-634.

NÚÑEZ, L.; NIELSEN, A. (ed.). Ruta: arqueología, historia y etnografía del tráfico sur Andino. Córdova: Encuentro Grupo Editor, 2011.

PINHEIRO-MACHADO, R. Caminos del contrabando. La fiscalización en el Puente de la Amistad y sus efectos en la cotidianeidad de la Triple Frontera. In: GIMÉNEZ, V.; MONTENEGRO, S. (ed.). La Triple Frontera: dinámicas culturales y procesos transnacionales. Buenos Aires: Espacio Editorial, 2010. p. 99-118.

PRADO, F. Los culebrones de la noche: un estudio antropológico del comercio informal de combustible en Tumbes. Ciencia y Desarrollo, Lima, v. 15, n. 2, p. 1-10, 2012. 
RABOSSI, F. En la ruta de las confecciones. Crítica en Desarrollo, Buenos Aires, n. 2, p. 151-171, 2008.

REEVES, M. Border work: spatial lives of the state in rural Cetral Asia. Ithaca: Cornell University Press, 2014.

RENOLDI, B. Narcotráfico y justicia en Argentina: la autoridad de lo escrito en el juicio. Buenos Aires: Antropofagia, 2008.

RENOLDI, B. Organización, crimen y acción. Relatos policiales de la Triple Frontera. In: BARREIRA, C.; SÁ, L.; AQUINO, J. (org.). Violência, ilegalismos e lugares morais. Campinas: Pontes, 2014. p. 479-507.

RENOLDI, B. Estados posibles: travesías, ilegalismos y controles en la Triple Frontera. Etnográfica, Lisboa, v. 19, n. 3, p. 417-440, 2015.

RIBEIRO, G. La globalización popular y el sistema mundial no-hegemónico. Nueva Sociedad, Caracas, n. 241, p. 36-62, 2007.

RICHARD, N. et al. El camino, el camión y el arriero: la reorganización mecánica de la Puna de Atacama (1930-1980). Historia 396, Valparaíso, v. 8, n. 1, p. 163-192, 2018.

ROSENBLITT, J. Centralidad geográfica, marginalidad política: la región de TacnaArica y su comercio, 1778-1841. Santiago: Centro de Investigaciones Diego Barros Arana, 2013.

SANTOS, M. Por otra globalización: del pensamiento único a la conciencia universal. Bogotá: Andrés Bello, 2004.

SARKAR, M. (ed.). Work out of place. Berlin: Walter de Gruyter, 2017.

SASSEN, S. Contrageografías de la globalización. Madrid: Traficantes de Sueños, 2003.

TASSI, N. et al. Hacer plata sin plata: el desborde de los comerciantes populares en Bolivia. La Paz: PIEB, 2013.

VEHLO, G. Accusations, family mobility and deviant behavior. Society for the Study of Social Problems, Knoxville, v. 23, n. 3, p. 268-275, 1976.

VELASCO, J. El azar de las fronteras: políticas migratorias, ciudadanía y justicia. Ciudad de México: FCE, 2016.

Recebido: 31/01/2019 Aceito: 24/06/2019 | Received: 1/31/2019 Accepted: 6/24/2019 